\title{
Automatic Extraction of Translations from Web-based Bilingual Materials
}

QIBo ZhU

Statistics Canada \&

Institute of Cognitive Science, Carleton University

Email:qzhu5@carleton.ca

DIANA INKPEN

School of Information Technology \& Engineering, University of Ottawa

Email:diana@site.uottawa.ca

AsH AsudeH

Institute of Cognitive Science \& School of Linguistics and Applied Language

Studies, Carleton University

Email:ash_asudeh@carleton.ca

\begin{abstract}
This paper describes the framework of the StatCan Daily Translation Extraction System (SDTES), a computer system that maps and compares webbased translation texts of Statistics Canada (StatCan) news releases in the StatCan publication The Daily. The goal is to extract translations for translation memory systems, for translation terminology building, for cross-language information retrieval and for corpus-based machine translation systems. Three years of officially published statistical news release texts at www.statcan.ca were collected to compose the StatCan Daily data bank. The English and French texts in this collection were roughly aligned using the Gale-Church statistical algorithm. After this, boundary markers of text segments and paragraphs were adjusted and the Gale-Church algorithm was run a second time for a more fine-grained text segment alignment. To detect misaligned areas of texts and to prevent mis-matched translation pairs from being selected, key textual and structural properties of the mapped texts were automatically identified and used as anchoring features for comparison and misalignment detection. Results show that SDTES is very efficient in extracting translations from Daily texts, and very accurate in identifying mismatched translations. With parameters tuned, the text-mapping part can be used to align officially published bilingual government web-site materials; and the text-comparing component can be applied in pre-publication translation quality control and in evaluating the results of statistical machine translation systems.
\end{abstract}

Key words: automatic translation extraction, bi-text mapping, machine translation, parallel alignment, translation memory system. 


\section{Introduction}

A rapidly growing body of research on natural language processing, machine translation and translation memory systems attests to the importance of automatic translation extraction. The applications of extracted translation pairs illustrate significant diversity. If the quality of translations is good and the translation pairs are well-aligned, the translation segments can represent a crucial resource for different natural language processing tasks (Moore, 2002; Gey et al., 2002) such as machine translation (Hutchins, 2005; Deng, Kumar \& Byrne, 2006; Simões \& Almeida, 2006) and cross-language information retrieval (Chen \& Gey, 2001). Extracted translation pairs can also be a useful reference for translation studies (Neumann \& Hansen-Schirra, 2005), computer-aided translation (Callison-Burch, Bannard \& Schroeder, 2005), and computer-assisted revision of translations (Jutras, 2000).

Translation pairs can be extracted from a variety of resources. Some of these resources are unannotated transcriptions of recorded meeting minutes, scanned copies of literary works, and data collections of text-only flat files. Some are resources with rich meta information, such as annotated bilingual corpora and bilingual hypertexts. Given the growing amount of bilingual documents on the web, it has come to the attention of researchers in computational linguistics that the web can be explored as a bilingual corpus (Resnik, 1999; Chen \& Nie, 2000). As such, the web is becoming an important source for extraction of translation pairs. Currently there is an increasing need for well-aligned translation pairs in natural language processing, machine translation and translation studies. For example, for corpus-based machine translation, quality translation pairs are in great demand as training data or translation templates. For many translation memory systems, the amount of raw feeds and the speed of generating them are not satisfactory. On the one hand, building annotated and well-aligned bilingual corpora to aid machine translation and human translation is time-consuming and difficult. On the other hand, we have so many officially translated pages on the web that are not explored for the purpose of bilingual corpus building. What we need is to seek ways to mine the widely available web resources to bridge the gap in the scarcity of bilingual data resources. Automatic translation extraction from web-based materials is one of the efforts in this direction. One obvious advantage of automatic translation extraction systems is the potential of enriching currently available electronic translation 
resources with limited manpower and lexicographical expertise (Tufiş, Barbu, \& Ion, 2004). In this paper, we describe one such translation extraction system --- the StatCan Daily Translation Extraction System (SDTES).

SDTES is a recently built system at Statistics Canada that attempts to amass, process, filter and format the translated texts from the webbased StatCan Daily data collection. There are two major components in the system: a text alignment component and a misalignment detection component. For the text alignment component, we designed a tworound procedure in adopting the Gale-Church statistical model for the alignment of web contents. Without this two-round procedure, using the Gale-Church length model to align the Daily HTML texts would have either become impossible or would have generated many false translation pairs. The SDTES misalignment detection component integrates different alignment techniques, and takes advantage of some important textual and structural information in HTML texts. It can help distinguish correctly aligned pairs that are true translations from those pairs that are misaligned and unusable. We developed a two-step cognate extraction method to generate cognate lists that could be used to assist the text alignment process for the purpose of detecting misaligned translation pairs. Results show that SDTES can generate rather clean translation pairs --- translation pairs that are of good quality and almost error free as far as alignment is concerned --- because the few problematic translation pairs can be automatically identified and eliminated. The precision and recall of the alignment mechanism and of the misalignment mechanism are very high. In addition, the text alignment algorithms and methods are straightforward, robust and can be easily applied to most of the government web materials in Canada where government websites have to present web materials in both English and French. The misalignment detection algorithm can help ensure the quality of aligned translation pairs and reduce greatly the repetitive manual tasks of verifying and proofreading the translation pairs before they become officially endorsed for use in machine translation systems, in translation memory systems and in other natural language processing applications.

Previous work in automatic translation extraction of web-based materials is mostly related to mining web contents as a bilingual corpus and aligning the bilingual texts. These are also the two primary challenges facing systems that extract translations from bilingual websites. As we know, the building of bilingual corpora can be traced back to the earliest translated texts, such as texts on the 196 B.C. 
Rosetta stone (Véronis, 2000b). However, the use of the web as a bilingual or multilingual corpus for language studies, for natural language processing and for cross-language information retrieval (Chen \& Nie, 2000; Resnik, 2003; Sigurbjörnsson, Kamps \& Rijke, 2005; Chesñevar, Sabaté \& Maguitman, 2006; Li \& Yang, 2006; Wang et al., 2006) is only in its infant stage. The relatively recent exploration of the web as a bilingual or multi-lingual corpus was made possible by the rapid growth in the number of web pages, and the availability of vast quantities of web-based translation texts involving many language pairs. Till now, the focus of most of the investigations in this field has been on the discovery and pairing of bilingual sites, domains, HTML documents and pages, although new research is emerging in processing and preparing HTML pages for the actual extraction of translation pairs when bilingual web pages are downloaded (Sanchez-Villamil et al., 2006). At the same time, extracting translations, whether from unannotated data resources or from meta-information-rich content, inevitably involves methods of aligning bilingual texts. To this end, various influential text alignment approaches (Gale \& Church, 1991; Brown, Lai \& Mercer 1991; Kay \& Röscheisen, 1993; Wu, 1994; Chen, 1996) --- whether statistical, lexical or hybrid --- were developed in the 1990s. However, they do not include ways to handle the particular features of HTML texts --- a text format that is increasingly popular. As a matter of fact, some features of HTML texts can be as important as the length of the sentence in the text alignment process. In this paper, we intend to investigate, through the description of the STDES system, the link between research in exploring the web as a bilingual corpus and research in the traditional parallel alignment approaches. We are interested in (1) how to mine web content and prepare HTML files for bilingual text alignment; (2) how to adopt one of the most influential alignment approaches, the Gale-Church statistical approach, for the efficient alignment of the web-based bi-texts; (3) how to integrate different alignment strategies with the structural features of HTML texts to aid the text alignment process and the automatic misalignment detection operation.

This paper is organized as follows. Section 2 gives a description of the StatCan Daily data sets used in SDTES. Section 3 discusses the SDTES methodology. It includes the pre-processing of the data, using the length-based statistical model to align the text segments, cognates extraction, detection of regions of possible alignment errors, and formatting of the aligned texts. In section 4, we highlight the SDTES 
results and the methods used for the evaluation of the results. Section 5 is the conclusion.

\section{Data sets}

The currently collected StatCan Daily data sets include English and French texts of Daily news releases of Statistics Canada from 2004 to 2006. The Daily is the flagship publication of Statistics Canada, and arguably the most important document collection of the agency. It is published officially at www.statcan.ca every working day, in two official languages. Usually Statistics Canada publishes an average of 4 to 5 Daily text releases in a day. The file names of the Daily HTML pages bear information about the language and date of publication. Release texts can contain expository texts, numerical tables, notes, graphs and note-to-reader text chunks. Some important major releases are long, running for pages, and some regular minor releases are short, containing only a few lines of texts.

Producing The Daily is a cooperative effort. There are people providing draft contents, people assembling and processing the texts and tables, people generating charts and graphs, people editing and polishing the texts, people checking the contents and styles, and people disseminating the finished documents. As there are many people working on The Daily on a daily basis, there are guidelines for the submission of release articles, templates for the recurring releases, rules for the usage of HTML markup, and procedures for quality-checking. Because there are dedicated professional editors working on the editing and translation of the Daily texts, the translations are mostly standard and consistent translations that are in agreement with the official publication guidelines for government departments and agencies in Canada. The HTML documents are formatted according to the common look and feel publishing standards for Canadian government websites. Normally, we can expect to find many correspondences in structural features like the use of cognates and HTML markup (Figure 1) in the English and French files.

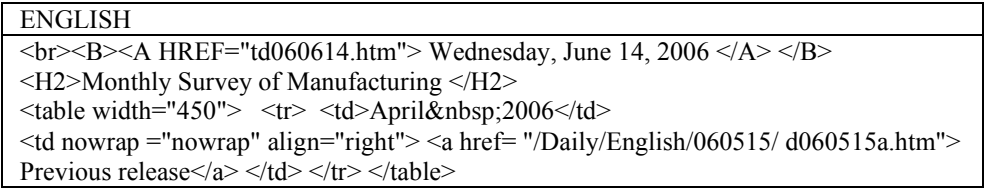




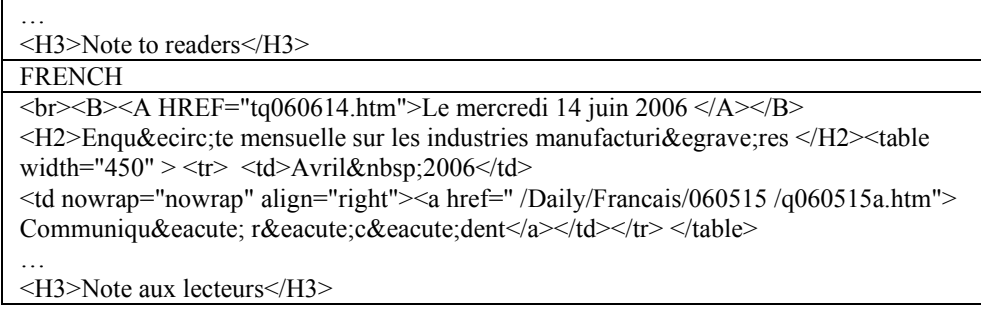

Figure 1. HTML source showing that the html structures in the two languages are very close to each other.

However, because of the writing conventions of different languages, there are sometimes structural differences in expressing the same content. This, as a result, can bring about differences in the use of HTML codes. For examples, in French, some characters in texts (such as the letter $o$ in the French abbreviation $n^{o}$ ) are in the superscript text format while they are not in that text format in English. In addition, because The Daily is also published in a PDF version, some charts, tables and text blocks can be put in floating positions to avoid graphs and tables crossing over PDF pages. This means that the floating elements such as graphs and tables can end up in different text positions in different languages. To keep the contents and particularly the positioning of the contents the same for the two publication versions (PDF and HTML), the floating nature of some charts, tables and text blocks in the PDF version is reflected in the corresponding HTML pages. For example, in Figure 2, the floating positioning of the "Note to readers" text block disrupts the order of paragraphs, and thus makes the alignment task significantly more difficult. 


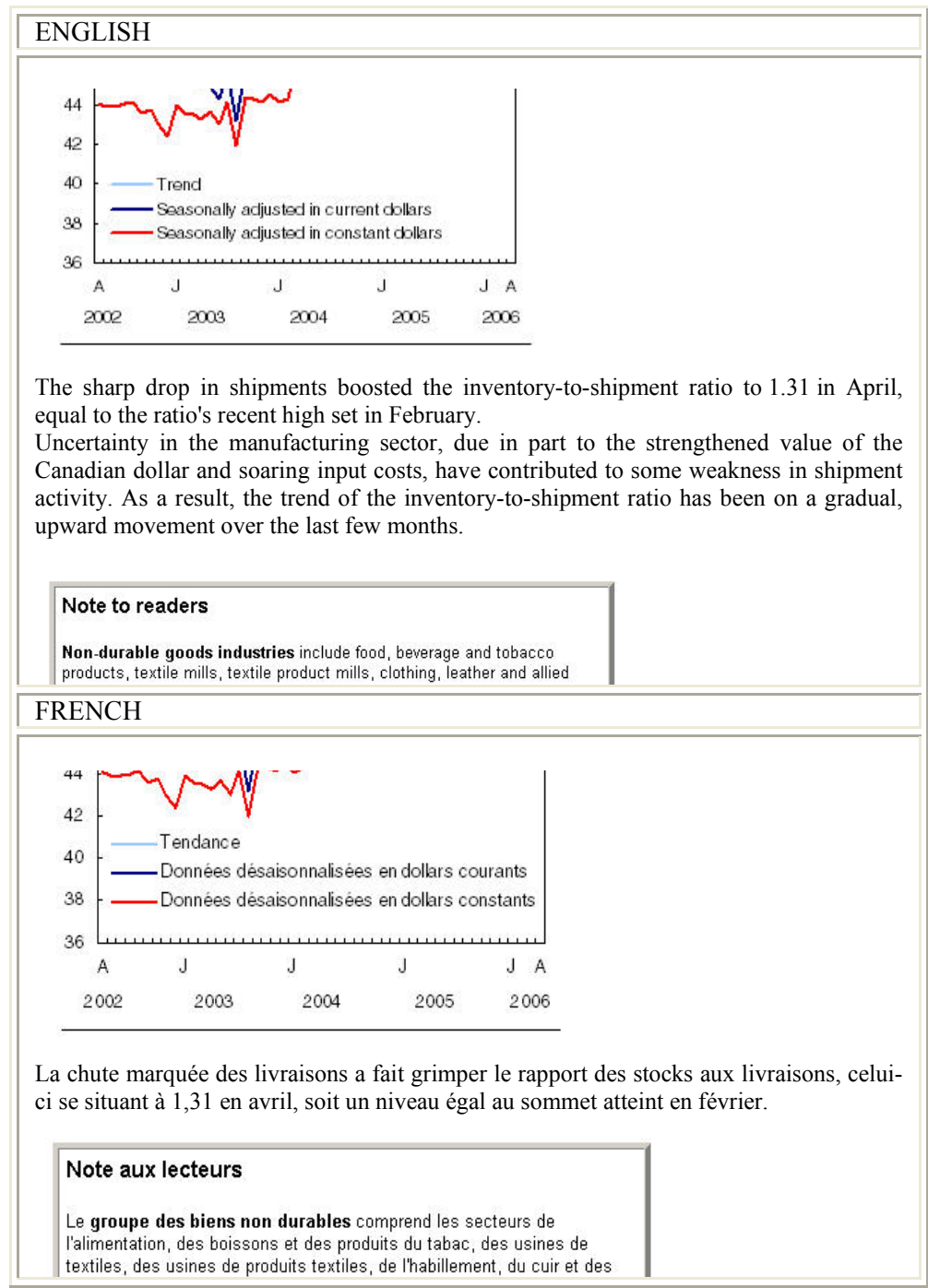

Figure 2. Floating text blocks disrupting the order of paragraphs as seen from the file set $060614 \mathrm{a}$. 
QIBO ZHU, DIANA INKPEN AND ASH ASUDEH

\section{Methodology}

The SDTES text alignment approach relies mainly on the Gale and Church algorithm (Gale and Church, 1991) of length-based statistical translation alignment. To enhance alignment accuracy and to eliminate manual intervention before alignment, the same statistical model is applied twice with different paragraph and sentence boundary definitions. For misalignment detection, we developed a two-step cognate extraction method. First, we used the K-vec algorithm (Fung and Church, 1994) for the initial word correspondence mapping. Then we designed the Acceptable Matching Sequence (AMS) search algorithm to generate cognate word lists that could serve as anchoring features for the detection of misalignment segments. SDTES was implemented in the Linux and Windows-Cygwin platforms with Perl and $\mathrm{C}$ as the major programming languages. Procedures and steps also included pre-alignment processing and post-alignment formatting.

\subsection{ASSEMBLING AND PRE-ALIGNMENT PROCESSING OF THE DATA SETS}

In collecting the data for SDTES, we directly accessed the archive storage area of The Daily. The file name scheme of The Daily reflects information about the language of the text and the publication date. For example, in the file name "d080108b.htm" $d$ stands for daily which means it is an English release. If this is a French release, it is $q$, representing the French word quotidien. 080108 is the date of publication and $b$ means that it is the second release for that day. We took only those files that matched the pattern d0[456] for English and $\mathrm{q} 0[456]$ for French in the file names. This enabled us to extract only those Daily releases that were published from 2004 to 2006. Since every Daily file was officially published on the Statistics Canada website, an alternative way to get the files is to simply grab the pages from http://www.statcan.ca/. Once all the data files were assembled, the next thing to do was to preprocess the HTML documents to pave the way for the use of the Gale-Church length-based alignment model. In our system, a text segment can be a sentence, two sentences together, a title, or a text unit in a table cell. For all the HTML files, the common header and footer wrappings were stripped. SDTES also does a conversion of some French coding so that most of the accent characters in French can be properly and consistently handled. One important task in the preprocessing stage was to re-organize some of the document's structures. As we have seen in section 2, The Daily release texts can contain floating tables, text chunks and graphs. For reasons of PDF 
pagination, some charts, graphs, tables and text blocks can chop off different numbers of paragraphs in between (see Figure 2). If texts were aligned as they were, the Gale and Church model could have generated many spurious and unusable translation correspondences. SDTES automatically re-organizes all the images, charts, graphs and tables, and moves the floating elements to the end of the document so that the perturbation of text segments is avoided.

\subsection{ALIGNMENT USING THE LENGTH-BASED STATISTICAL MODEL}

For the text alignment component of SDTES, we use the Gale and Church statistical alignment model (Gale and Church, 1991). The basic assumption of the Gale and Church length-based algorithm is that there is a strong likelihood that a long sentence in English will correspond to a long sentence in French; similarly a short sentence in English will correspond to a short sentence in French. Roughly speaking, if the average lengths of sentences in French and English are known, it is possible to set up a distribution of alignment possibilities from the sentence length information.

For a text in English to be matched up with a text in French, there are always a number of different possible alignments. Given parallel HTML texts $E$ (for English) and $F$ (for French), an alignment $A$ is a segmentation of $E$ and $F$ into $L_{x} \Leftrightarrow L_{y}$ pairs, where each $L_{x}$ is an English segment, $L_{y}$ is a French segment, and $L_{x}$ and $L_{y}$ are mutual translations. The goal is to find the maximum likelihood alignment, given a pair of texts:

$$
\arg \max _{A} \operatorname{Pr}(A \mid E, F)
$$

The first approximation is that the probability of any aligned segment pair in an alignment is independent of any other segment pair:

$$
\operatorname{Pr}(A \mid E, F) \approx \prod_{\left(L_{X} \Leftrightarrow L_{Y}\right) \in A} \operatorname{Pr}\left(L_{X} \Leftrightarrow L_{Y} \mid E, F\right)
$$

The second approximation is that each $\operatorname{Pr}\left(L_{X} \Leftrightarrow L_{Y} \mid E, F\right)$ does not depend on the contents of the specific HTML texts, but depends only on the contents of the segments within the alignment, so that the $\operatorname{Pr}\left(L_{X} \Leftrightarrow L_{Y} \mid E, F\right)$ in the above formula becomes $\operatorname{Pr}\left(L_{X} \Leftrightarrow L_{Y} \mid L_{X}, L_{Y}\right)$. If we think that the only features that can influence the probability of 
the alignment are the length paradigms, given $s_{l}=\operatorname{length}\left(L_{x}\right)$ and $s_{2}=$ length $\left(L_{y}\right)$, then we can have: $\operatorname{Pr}\left(L_{X} \Leftrightarrow L_{Y} \mid s_{1}, s_{2}\right)$ instead. Next, we convert the maximization of the approximation into a minimum-sum problem, and implement the minimization by dynamic programming:

$$
\begin{aligned}
& \arg \max _{A} \operatorname{Pr}(A \mid E, F) \approx \arg \max _{A} \prod_{\left(L_{X} \Leftrightarrow L_{Y}\right) \in A} \operatorname{Pr}\left(L_{X} \Leftrightarrow L_{Y} \mid L_{X}, L_{Y}\right) \\
& =\arg \min _{A} \sum_{\left(L_{X} \Leftrightarrow L_{Y}\right) \in A}-\log \operatorname{Pr}\left(L_{X} \Leftrightarrow L_{Y} \mid L_{X}, L_{Y}\right)
\end{aligned}
$$

The Gale-Church algorithm accepts input from two text files at a time: one text file for each of the languages. The preparation of the text files involves (1) breaking the text files into lines of words, one word per line and (2) adding two types of place-holding markers: one for the end of the paragraph (.EOP) and one for the end of the sentence (.EOS). For the application of the Gale-Church model, the actual sentenceending or paragraph-ending markers such as .EOS and .EOP can take arbitrary names, but the number of paragraph-ending markers (.EOP) in the English file should be equivalent to the number of paragraph-ending markers in French. If the paragraph numbers are different, the program will not proceed. The variance in the number of sentences (or .EOS) will not matter as much because in aligning sentences, we can have different matching types such as 1:2, 2:1, 1:0 and 0:1.

It is not uncommon to find a discrepancy in the number of paragraphs in The Daily document pairing process. Usually the gap is not great. Nevertheless, as noted above, a slight difference of one or two can bring the alignment process to a halt. It would be rather timeconsuming to manually identify the exact places where the end-ofparagraph markings are missing, or are to be inserted. To tackle this problem, we defined the two types of boundary marking symbols in the first round of text alignment as follows:

EOA is a paragraph pseudo-boundary marker that indicates a block of text containing, in most cases, a series of paragraphs separated by a main HTML element. In the first round of alignment, an .EOA marked text block is treated as a "large" paragraph.

.EOP is a sentence pseudo-boundary marker that actually indicates the end of a paragraph. An .EOP marked paragraph, though it usually 
contains more than one sentence, is considered a "large" sentence in the first round of text alignment.

In SDTES, a limited number of HTML tags that can be used to mark the beginning of a text block are categorized as the main HTML elements. They include tags such as title, table, h1, h2, h3. The first round of text alignment in SDTES is at the macro level. STDES first counts these few main HTML elements to see if the pair of text files has the same number of main HTML features in them. If the numbers were the same, the system splits the texts into blocks separated by these feature HTML tags. After this, the system marks these text blocks using the paragraph pseudo-boundary symbol .EOA, and marks the original paragraph ending places using the sentence pseudoboundary marker .EOP. If the numbers of major HTML elements are different, the system treats the whole text document as a single pseudo paragraph and the original paragraphs as pseudo sentences. So the parameters for the first implementation of the Gale-Church algorithm are:

align -D '.EOA' -d '.EOP' filename_eng filename_fre

An obvious advantage of doing the macro level alignment based on the main HTML elements is that by introducing the HTML structural information into the statistical method, we can avoid misalignment across text blocks. Even if there are misalignments, they will be kept within a minimum text region.

To do the second round of alignment, SDTES automatically reconstructs the English document and the French document from the aligned paragraphs of the output file. When the first round of text alignment has been completed using the length-based model, the alignment results are put in a file ending in .al with translation text blocks containing paragraphs clearly indicated. However, to realign the texts, the aligned pairs have to be separated into two files: one for the English texts, and the other for the French texts. So, the texts in different languages were reassembled and new definitions were given to the two types of boundary markers:

EOP marks the end of each aligned text block in the output file that is generated as a result of the first round of alignment. In most cases it contains one paragraph, but in some cases it can contain two paragraphs. 
EOS is the text segment ending symbol in the second round of text alignment. It marks the end of a text segment which in most cases includes one sentence.

By reorganizing the text structures on the basis of the paragraph pairing results in the first around of text alignment, SDTES was able to reset the English and French documents to the original text format prior to the initial alignment. The two input files are processed with the newly assigned boundary symbols .EOP and .EOS:

\section{align -D '.EOP' -d '.EOS' filename_eng filename_fre}

Figure 3 contains some examples that are produced in the second round of text alignment in SDTES. They are results of text alignment at a more fine-grained text segment level. We also call the text segment unit that is aligned with its translation counterpart in each of the translation pairs "a bead of text".

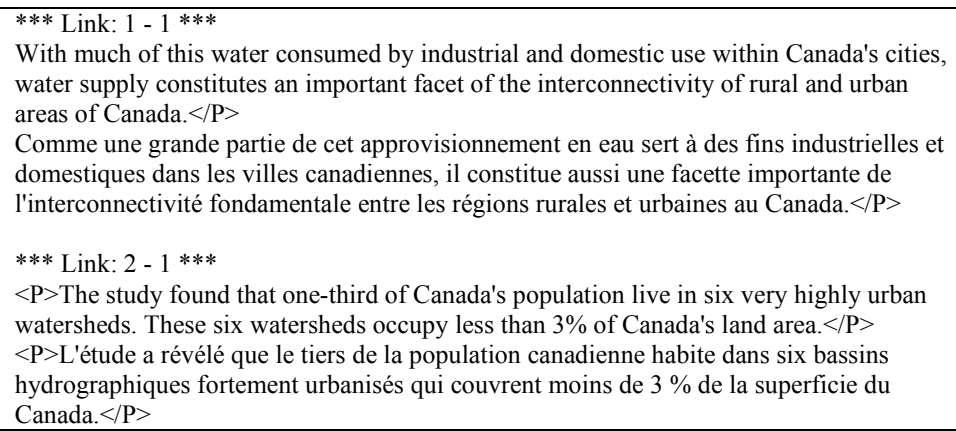

Figure 3. Examples of aligned text segments from $060105 \mathrm{~b}$.

\subsection{COGNATES EXTRACTION BASED ON THE K-VEC ALGORITHM AND} ACCEPTABLE MATCHING SEQUENCE SEARCH

Since Simard et al. (1992) suggested to use cognates in the alignment of bi-texts, different definitions and ways of extracting and measuring cognates have been proposed (Melamed, 1999; Danielsson \& Muhlenbock, 2000; Ribeiro et al., 2001; Inkpen, Frunza \& Kondrak, 2005). In the parallel alignment context, cognates are not necessarily words with etymological ties. They can be identical or graphically similar occurrences in the two languages (Véronis, 2000a). 
In the current system, we define cognates as words that share a good portion, either interrupted or uninterrupted, of the character string in a reasonably computable search space. This means that cognates should be "more or less like each other in form" (McArthur, 1992) and that for two words to be considered a cognate pair, they should occur in approximately the same text region. Since our goal of finding cognates is to use them to assist parallel text alignment in the process of misalignment detection, we require that two cognate words appear in text regions of reasonably computable search space. If a source word in French appears in paragraph 3, but the target English word is in paragraph 10 , even if they are a cognate pair, they are not recruited in our cognate list. This is because the two words in this case are not within a "reasonably computable search space" and we do not want two widely separated sentences to be aligned as a translation pair. In addition, the matching portion requirement varies with the maximum length of the words involved. For example, if the French word has 11 characters, we require that the matching English word to be within the length range of $11 \pm 3$. What is more, at least 8 characters in two substrings in the French word should match two corresponding substrings in the English word in the correct order.

The first step in SDTES cognate extracting algorithm is to produce candidate cognate lists using the K-vec algorithm. The goal is to find cognate candidates within an acceptable text region range or computable search space and limit the number of words to be considered as cognate pairs. The K-vec method was developed by Fung and Church as a means of generating "a quick-and-dirty estimate of a bilingual lexicon" that "could be used as a starting point for a more detailed alignment algorithm ..." (Fung and Church, 1994). The assumption is that if two words are translations of each other, they are likely to occur almost an equal number of times in approximately the same region in the parallel texts. In trying to find word-to-word translations, K-vec does not require any prior knowledge of sentence boundaries. The algorithm splits each text to be aligned into $K$ subsections or pieces. Although the $K$ value can be chosen and adjusted, care should be taken not to make it too large or too small. If $K$ is very large, the total number of words in each piece would be small and we may have the risk of missing translations. If $K$ is very small, the number of words in each piece would be large and we lose the advantage of dividing the text into pieces for the purpose of locating a word and its translation in corresponding pieces. Fung and Church 
corresponding $K$ piece. This is one of the reasons why, in the candidate cognate list in Figure 4, we see so many noisy pairs that are not cognates. As soon as the rough candidate cognate list is produced, there is a filtering device in SDTES to keep only those pairs that have the last three numbers in the output line (such as industrielle $<>$ industrial $<>12$ 2 ) very close to each other. The three numbers indicate the corresponding $K$ pieces where the French word and the English word can be found, the $K$ pieces containing the French word and the $K$ pieces in which the English word occur. In this way, spurious correspondences such as octobre $<>$ several $<>1102$ or secteur $<>$ automotive $<>1112$ can be eliminated before candidate pairs of cognates are further processed.

The second step in the extraction of cognate pairs is to apply our pattern matching algorithm, the Acceptable Matching Sequence (AMS) search. An AMS has two non-overlapping substrings that can be matched in the same order in both of the words in a cognate pair. The algorithm extracts two substrings $\left(\theta^{a}\right.$ and $\left.\beta^{b}\right)$ from a source word, say a French word $\left(W_{l}\right)$, with a length threshold $(T)$ for the two substrings combined. Then it searches for the string sequence that contains the two substrings in the same order in the target English word $\left(W_{2}\right)$. Gaps or "Don't Care Characters" (DCCs) are acceptable before, after or between the two substrings. The initial value $a$ in $\theta^{a}$ is set to 0 , and $b$ in $\beta^{b}$ to $T$. If a match does not occur, one substring $\theta^{a}$ is increased from left to right $(a=a+1)$ while the other substring $\beta^{b}$ gets decreased in the same direction $(b=b-1)$. The search continues till a two-substring match is found or $a>T$ or $b<0$. An AMS can be defined as in the following regular expression.

$$
W_{l}=(.)^{*} \theta^{a}(.)^{\{0, c\}} \beta^{b}(.)^{*}
$$

Where:

(.)* is a substring of any character combinations. This substring can also be an empty substring.

(. $)^{\{0, c\}}$ is a substring of 0 to $c$ characters. $0<c<4$.

$\theta^{a}$ is the first substring to be matched in $W_{2}$. The length of this substring is $a$.

$\beta^{b}$ is the second substring to be matched in $W_{2}$. The length of this substring is $b$.

$T$ is the combined length of $\theta^{a}$ and $\beta^{b} .0 \leq a \leq T, 0 \leq b \leq T, a+b=T$. 
$x \leq \operatorname{length}\left(W_{1}\right) \leq y$ or $x \leq \operatorname{length}\left(W_{2}\right) \leq y$, | length $\left(W_{1}\right)-$ length $\left(W_{2}\right) \mid<z, 0 \leq z \leq 4$.

The AMS search model can be demonstrated as in Figure 5.

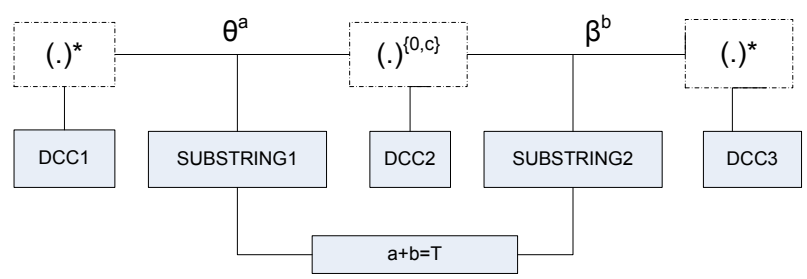

Figure 5. AMS search model for $x \leq$ length $\left(W_{l}\right) \leq y$. $W_{1}$ becomes an AMS only when the two substrings $\left(\theta^{a}\right.$ and $\left.\beta^{b}\right)$ are both matched in the correct order in $W_{2}$. DCC1, DCC2, DCC3 are "Don't Care Characters". They don't need to be matched in $W_{l}$ and $W_{2}$.

For example, if the length of the longer string of two words $\left(W_{l}\right.$ and $W_{2}$ ) is 9 and the length difference between the two words is 3 , we set $T=7$, and $c=2$. Figure 6 represents one of the "worst-case" AMS search problems in SDTES. Here, we want to find cognates from two candidate words of which the longer one has a length of more than 10 characters. We set the minimum combined matching length requirement $T$ as 8 . The two substrings can be separated by 0 to 3 characters $(c=3)$. The length difference between the two words is less than $4\left(\mid \operatorname{length}\left(W_{l}\right)\right.$ - length $\left.\left(W_{2}\right) \mid<z ; z=4\right)$. In the solution to the AMS search problem, we are interested in finding if the properties of the two substructures are shared in the both of the words $W_{1}$ and $W_{2}$. If the two substrings $\theta^{a}$ and $\beta^{b}$ are exactly the same in the two words, and they occur in $W_{l}$ and $W_{2}$ in the same order, we say $W_{1}$ and $W_{2}$ are cognates. Figure 6 shows the AMS search process.

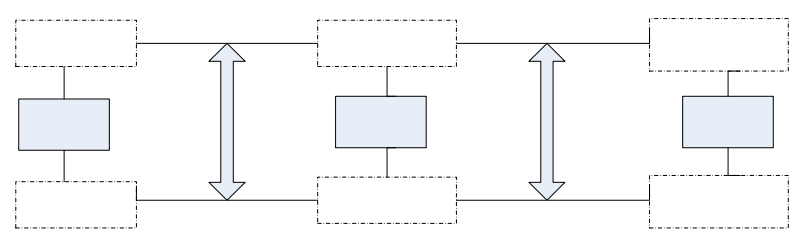


sequence. Once a two-substring match is found, the search stops. There is no need to do cost calculations of insertion or deletion to get the minimum edit distance. The algorithm does not have to traverse all the components of the patterns to find, calculate and compare all the possible matching sequences to identify the longest common sequence. This can reduce the complexity of computational operations. Also, by focusing on only those pairs that can help with the text alignment, the algorithm is able to filter out many candidate pairs that are either not genuine translation correspondences or true cognates that could not help the text alignment prior to the actual substring matching process. Therefore, the AMS search model is easy to use and efficient in achieving our purpose.

\subsection{DETECTION OF REGIONS OF POSSIBLE ALIGNMENT ERRORS}

As stated in the description of the data sets, translations in the published Daily releases are mostly clean and consistent translations in agreement with the Canadian guidelines of publications for government websites. Translation errors such as deletion and insertion are rare. But it is possible that the correct translation pairs are misaligned. In SDTES, we developed an algorithm to detect the regions of possible alignment errors. Figure 8 is the alignment detection operation diagram. It shows the major steps involved in arriving at one of the two outcomes: pass and problem. The detecting process starts from two prior filtering mechanisms. One of them is the length ratio criteria. If a text segment in one language is more than 3 times longer than the corresponding text segment in the other language, the pair is marked as a problem pair. The second criterion is matching type. Because the extracted translations are independent translation pairs that will be used for translation memory systems and cross-language information retrieval systems, matching types like 1:0 and 0:1 have to be discarded. When these two criteria have been checked, SDTES compares the structural and lexical clues of the HTML text segments for further detection. These clues include selected cognates, punctuations, numbers and HTML tags. 


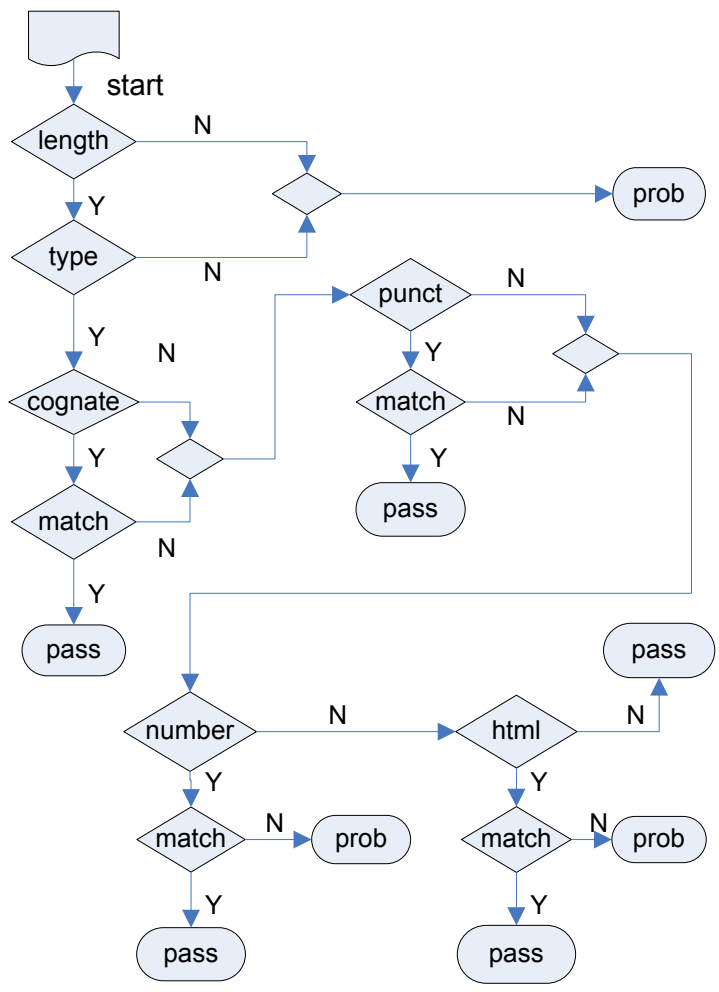

Figure 8. Text alignment problem detection framework

For the cognates, we use the list of words that are automatically extracted by the algorithms we described above. When SDTES accepts an aligned pair as input in the misalignment detection operation, it scans through the input text segments to see if there are cognates present. If there are, then the system compares them to see if they match in the texts of two languages. If a match is found, the two text segments are a good translation pair. Otherwise, the candidate pair of text units is passed to the next step of detection. For punctuation, SDTES mainly maps the correspondences using comma, plus sign and minus sign, parenthesis, colon and semi-colon, etc. If the system identifies the equivalence in the use of punctuations (frequency of occurrence $n>0$ ), it marks the pair as pass. We assume that, in the statistical news release 
texts, numbers are literally translated. If we have numbers in the English text, normally we should have numbers in the corresponding French text segment. If most of the numbers in the candidate pair do not match, the decision is that there might be a problem in alignment or in translation. When the system does not find any numbers in the aligned text segments, it continues the detection by looking for markups of HTML coding. The distinguishing HTML markups SDTES uses include $\langle\mathrm{h} 2\rangle\langle\mathrm{h} 3\rangle\langle i\rangle\langle\mathrm{b}\rangle\langle\mathrm{a} \ldots\rangle\langle$ table $\rangle$ and others. We did some HTML style unification formatting so that some parts of the HTML codes are highlighted, while some are ignored. For example, the code $<$ a $\ldots>$ becomes $<$ a alink $>$ after the unification formatting. Once the system gathers the key HTML structural features in the aligned texts, a comparison is done to see if the HTML tags are the same. If they are, the segments are a good aligned pair of translations; and if they are not, the system marks it as a problematic alignment region. Finally, we have the zero-tolerance principle: if there are no structural and textual clues present, and if the two prior filtering criteria (length and matching type) are checked, we mark the segment as pass. Here is a sample of the misalignment detection results list (Figure 9):

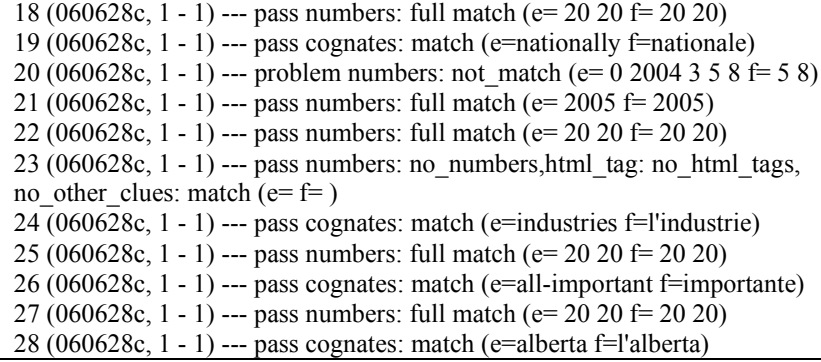

Figure 9. Sample of misalignment detection. 'Pass' means the alignment is identified as correct, and 'problem' means the pair is a misalignment.

\subsection{POST-ALIGNMENT FILTERING AND FORMATTING}

We added a filter on the list of stored translations to eliminate aligned pairs such as:

1. Pairs that contain pure meta information coding or codes that are derived from the HTML coding unification process.

$* * *$ Link: $1-1 * * *$

$<$ hr size $=1><$ zcorpus text $=10><$ zcorpus table $=10><$ hpara $=$ e35:f35 $>$ 
$<$ hr size $=1></$ zcorpus text $=10><$ zcorpus table $=10><$ hpara $=$ e35:f35 $>$

(040107a)

2. Alignment segments that include only the numerical information:

*** Link: $1-1 * * *$

2001/02 <hpara $=$ e35:f35>

2001-2002 <hpara $=$ e35:f35>

(040107a)

3. Only one pair is kept for duplicate patterns and constructions that are repeated frequently in the collection of texts:

*** Link: $1-1 * * *$

Retail trade $</$ TITLE $>$

Commerce de détail $</$ TITLE $>$

(060821a)

*** Link: $1-1 * * *$

$<\mathrm{p}><\mathrm{B}>$ Definitions, data sources and methods: survey numbers, including related surveys, $<$ a alink $>2406</ \mathrm{A}>$ and $<$ a alink $>2408</ \mathrm{A}>.</ \mathrm{B}></ \mathrm{P}>$

$<\mathrm{p}><\mathrm{B}>$ Définitions, source de données et méthodes : numéros d'enquête, $\mathrm{y}$ compris ceux des enquêtes connexes, $<$ a alink $>2406</ \mathrm{A}>$ et $<$ a alink $>2408$

$</ \mathrm{A}>.</ \mathrm{B}></ \mathrm{P}>$

(060821a)

These aligned segments were further cleaned and organized in the XML format for easy exportability into the translation memory system, information retrieval database systems, and other application systems. Meta information items about each of the aligned pairs were recorded such as the string length information (before the HTML codes are stripped), the source of the matched strings, the matching patterns (1:1, $1: 2,2: 1,2: 2$ ), and misalignment detection result (pas for pass and pro for problem). The SDTES output format (see Figure 10 for a modified version) is intended to serve as an intermediary form from which different user-friendly formats for different applications can be derived. The interim format is good for use with UNIX system tools and Perl one-liners for quick finding of translations, as well as for various sorting, analyzing and statistical tasks. It can be easily converted into different feeding formats in different systems such as Daily translation recycling templates system (text block based format), translation memory systems (format with texts of different languages assembled in different files), bilingual information retrieval (format required by the search engine), and bilingual text navigation interface (format required by the field specifications of the SQL database). The conversion from the basic STDES format to the required input formats of different 
systems can be done with a few lines of coding or, in some cases, in a text editor environment. Figure 10 includes some examples of final aligned segments with the English part beginning with 'Wholesale trade activity'. For the sake of presentation clarity, line breaks are added to different levels of XML elements. 


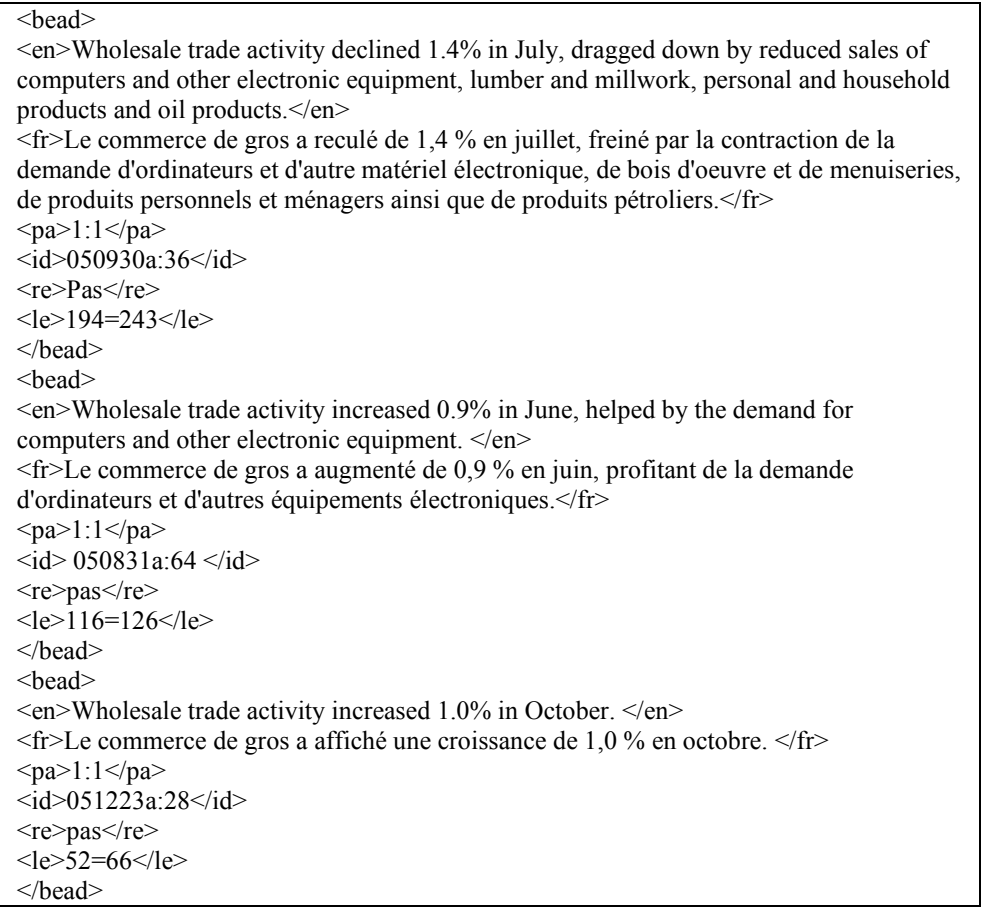

Figure 10. Modified SDTES format for aligned segments for English texts beginning with 'Wholesale trade activity'. In the original SDTES format, only the tag $</$ bead $>$ takes line breaks.

\section{Evaluation and results}

To evaluate the performance of the alignment, we need a reference collection of manually aligned text segments. The aligned pairs are supposed to be correct in this reference collection. Then we can measure how the machine aligned pairs differ from those in the reference collection.

Let $M$ be the set of segments in the manually aligned reference collection, $A$ be the set of machine aligned segments and $C$ be the set of correctly aligned segments, precision $(P)$ and recall $(R)$ can be defined as follows: 


$$
P=\frac{|A \cap C|}{|A|} \quad R=\frac{|A \cap M|}{|M|}
$$

For evaluation, we randomly chose one aligned file for every two months. The same file is manually aligned for the building of the reference collection. Then we checked the machine aligned pairs to arrive at the number of correctly aligned segments. For the 18 aligned files used for evaluation, the average alignment precision is 0.96 and recall is 0.97 (see Table I). This compares favorably with the precision and recall values of most of the other alignment classifiers.

\begin{tabular}{|l|l|l|l|l|l|l|l|}
\hline File & $\begin{array}{l}\text { Machine } \\
\text { aligned } \\
\text { pairs (A) }\end{array}$ & $\begin{array}{l}\text { Correctly } \\
\text { aligned pairs } \\
\text { in A (C) }\end{array}$ & $\begin{array}{l}\text { Manually } \\
\text { aligned } \\
\text { pairs (M) }\end{array}$ & $\begin{array}{l}\text { A } \\
\text { intersect } \\
\text { M }\end{array}$ & $\begin{array}{l}\text { Recall } \\
(\mathrm{R})\end{array}$ & $\begin{array}{l}\text { Precision } \\
(\mathrm{P})\end{array}$ \\
\hline $040213 \mathrm{~b}$ & 214 & 212 & 214 & 212 & 0.99 & 0.99 \\
\hline $040415 \mathrm{a}$ & 109 & 103 & 109 & 103 & 0.94 & 0.94 \\
\hline $040611 \mathrm{~b}$ & 92 & 83 & 94 & 90 & 0.96 & 0.90 \\
\hline $040715 \mathrm{e}$ & 17 & 15 & 16 & 15 & 0.94 & 0.88 \\
\hline $041015 \mathrm{c}$ & 11 & 11 & 11 & 11 & 1 & 1 \\
\hline $041223 \mathrm{a}$ & 100 & 100 & 100 & 100 & 1 & 1 \\
\hline $050107 \mathrm{a}$ & 119 & 119 & 119 & 119 & 1 & 1 \\
\hline $050414 \mathrm{a}$ & 105 & 105 & 105 & 105 & 1 & 1 \\
\hline $050629 \mathrm{~b}$ & 72 & 72 & 72 & 72 & 1 & 1 \\
\hline $050721 \mathrm{a}$ & 121 & 121 & 120 & 119 & 0.99 & 1 \\
\hline $050922 \mathrm{a}$ & 116 & 116 & 116 & 116 & 1 & 1 \\
\hline $051109 \mathrm{a}$ & 185 & 185 & 190 & 180 & 0.95 & 1 \\
\hline $060111 \mathrm{a}$ & 86 & 84 & 87 & 85 & 0.98 & 0.98 \\
\hline $060308 \mathrm{~b}$ & 48 & 48 & 48 & 48 & 1 & 1 \\
\hline $060608 \mathrm{~d}$ & 31 & 29 & 34 & 32 & 0.94 & 0.94 \\
\hline $060822 \mathrm{~b}$ & 57 & 48 & 58 & 51 & 0.88 & 0.84 \\
\hline $061005 \mathrm{~b}$ & 117 & 117 & 117 & 117 & 1 & 1 \\
\hline $061213 \mathrm{e}$ & 17 & 15 & 16 & 14 & 0.88 & 0.88 \\
\hline & & & & & & \\
\hline Average & & & & 0.97 & 0.96 \\
\hline
\end{tabular}

Table I. Alignment evaluation parameters

The task of the misalignment detection algorithm is to traverse every pair of aligned segments to see if they are indeed a correctly aligned pair. It can happen that the aligned pair is a perfect translation 
of each other, but the detection algorithm wrongly labels the pair as a misaligned one, or a misaligned pair can be detected as a pair of perfect translations and the algorithm fails to capture the misalignment. In evaluating the misalignment detection algorithm, we used the same files that were randomly chosen for the evaluation of the aligning algorithm. We define precision and recall as before,but interpret the comparison sets $A, C$, and $M$ differently.

Here, we focus on the aligned pairs that are "perfect translations (PTs) of each other". $M$ is the reference, i.e. the number of aligned PTs that the human evaluator thinks the misalignment detection algorithm should have reported or proposed. $A$ refers to the number of aligned pairs that the algorithm proposed as PTs. $C$ is the number of aligned pairs that are correctly reported as PTs by the detection algorithm. The recall represents the proportion of algorithm-proposed PTs $(A)$ that are right with respect to the reference $(M)$, and the precision is the proportion of correctly proposed PTs $(C)$ with respect to the total of those proposed $(A)$. From the results shown in Table II, we can see that the system is accurate in identifying correctly aligned translation pairs $(\mathrm{P}=.998$ and $\mathrm{R}=.996)$.

\begin{tabular}{|l|l|l|l|l|l|l|}
\hline File & $\begin{array}{l}\text { Machine } \\
\text { identified } \\
\text { matching } \\
\text { pairs (A) }\end{array}$ & $\begin{array}{l}\text { Correctly } \\
\text { identified } \\
\text { matching } \\
\text { pairs in A } \\
\text { (C) }\end{array}$ & $\begin{array}{l}\text { Manually } \\
\text { identified } \\
\text { matching } \\
\text { pairs (M) }\end{array}$ & $\begin{array}{l}\text { A } \\
\text { intersect } \\
\text { M }\end{array}$ & $\begin{array}{l}\text { Precison } \\
\text { (P) }\end{array}$ & $\begin{array}{l}\text { Recall } \\
\text { (R) }\end{array}$ \\
\hline 040213b & 212 & 211 & 212 & 211 & 0.995 & 0.995 \\
\hline $040415 \mathrm{a}$ & 105 & 102 & 102 & 102 & 0.971 & 1 \\
\hline $040611 \mathrm{~b}$ & 78 & 78 & 79 & 78 & 1 & 0.987 \\
\hline $040715 \mathrm{e}$ & 15 & 15 & 15 & 15 & 1 & 1 \\
\hline $041015 \mathrm{c}$ & 11 & 11 & 11 & 11 & 1 & 1 \\
\hline $041223 \mathrm{a}$ & 100 & 100 & 100 & 100 & 1 & 1 \\
\hline $050107 \mathrm{a}$ & 118 & 118 & 119 & 118 & 1 & 0.992 \\
\hline $050414 \mathrm{a}$ & 105 & 105 & 105 & 105 & 1 & 1 \\
\hline $050629 \mathrm{~b}$ & 72 & 72 & 72 & 72 & 1 & 1 \\
\hline $050721 \mathrm{a}$ & 121 & 121 & 121 & 121 & 1 & 1 \\
\hline $050922 \mathrm{a}$ & 116 & 116 & 116 & 116 & 1 & 1 \\
\hline $051109 \mathrm{a}$ & 183 & 183 & 185 & 183 & 1 & 0.989 \\
\hline $060111 \mathrm{a}$ & 84 & 84 & 84 & 84 & 1 & 1 \\
\hline $060308 \mathrm{~b}$ & 47 & 47 & 48 & 47 & 1 & 0.979 \\
\hline $060608 \mathrm{~d}$ & 27 & 27 & 27 & 27 & 1 & 1 \\
\hline $060822 \mathrm{~b}$ & 49 & 49 & 49 & 49 & 1 & 1 \\
\hline
\end{tabular}




\begin{tabular}{|l|l|l|l|l|l|l|}
$061005 \mathrm{~b}$ & 116 & 116 & 117 & 116 & 1 & 0.991 \\
\hline $061213 \mathrm{e}$ & 15 & 15 & 15 & 15 & 1 & 1 \\
\hline & & & & & & \\
\hline Average & & & & & 0.998 & 0.996 \\
\hline
\end{tabular}

Table II. Misalignment detection evaluation parameters.

All in all, SDTES assembled a total of 3,874 documents for each language (English and French) to compose the StatCan bilingual data collection. The collected web documents were published over a period of 736 days, with an average of around 5 release texts a day (the actual number is 5.264). This bilingual Daily data collection has 1,611,230 running words for English and 2,050,948 running words for French. The word translation ratio of French to English is 1.3:1, which means that for every ten English words, we use 13 French words in the StatCan Daily texts. Using the Gale and Church length-based model, all the text segments were aligned with one of the 6 alignment patterns: $0: 1,1: 0,1: 1,1: 2,2: 1,2: 2$. The most common pattern is $1: 1(\mathrm{p}>.95)$ indicating that more than $95 \%$ of the text segments are sentence to sentence translations. There are examples of paraphrasing (patterns 2:1, 1:2 and 2:2), but deletions and insertions (patterns 1:0 and 0:1) in translation are rare (see Table III).

\begin{tabular}{|l|l|l|l|l|l|l|}
\hline $\begin{array}{l}\text { Alignment } \\
\text { pattern }\end{array}$ & $1: 1$ & $1: 2$ & $2: 1$ & $0: 1$ & $2: 2$ & $1: 0$ \\
\hline Frequency & 92,495 & 2997 & 1307 & 9 & 112 & 22 \\
\hline Percentage & 0.9541 & 0.0309 & 0.0135 & 0.0001 & 0.0012 & 0.0002 \\
\hline
\end{tabular}

Table III. Alignment types for the StatCan data collection. Here aligned text segments may include lines of pure HTML markup or other meta-information codes.

SDTES parsed all the aligned text segments and used key features such as numbers, HTML markups and cognates to judge if there were possible alignment errors in each of the aligned pairs. 96,283 translation pairs passed the alignment check, and a total of 659 problematic aligned segments were detected. The number of misaligned pairs that escaped the detection was minimal. Although there were examples of false misalignment, more than $99 \%(\mathrm{P}=.998)$ of the identified correct alignment pairs are truly reliable translation pairs. After filtering and formatting, 70,555 aligned segment pairs were generated and they are ready to be used in many applications and systems such as translation 
memory systems, information retrieval templates, natural language processing databases, and machine translation systems.

While our methodology has achieved good outputs (alignment precision $\mathrm{P}>.96$ ) for the domain-specific bilingual text data, there are also limitations. When we examine the aligned pairs, we still find some examples, although the number is very small, of chains of misaligned sentences caused by the swapping of positions in the translation texts. For example, if in a translation document pair, the $35^{\text {th }}$ text segment in English $\left(L_{X 35}\right)$ is the translation of the $40^{\text {th }}$ text segment in French $\left(L_{Y 40}\right)$, although the translation is right, it can amass misaligned segment pairs because the translation spans beyond more than 3 text segments, and the Gale and Church algorithm cannot handle it.

Although the StatCan Daily release texts in the bilingual text collection are suitable for use of the Gale-Church algorithm, some short sentences on only one side of the alignment pairs which carry no discriminative feature information can be a source of misalignment. Judging by the length-based alignment criterion, appending the short sentence to the previous sentence or combining the short sentence with the following sentence would not make much difference. This increases difficulty in alignment.

When examining the SDTES-generated cognate lists word by word, we found some misclassified pairs (see table IV). The false friends are mostly false positives caused by accidental similarity in the orthographic form. They are likely to be on the lists of any cognate classifier that depends on measures of orthographic similarity.

\begin{tabular}{|l|l|l|l|}
\hline French & English & French & English \\
\hline consiste & considers & entre & tenure \\
\hline cours & court & estivaux & festival \\
\hline abordable & affordable & fiscale & scale \\
\hline aller & smaller & finlande & mainland \\
\hline exercer & exerted & grands & brands \\
\hline fiable & reliable & mains & gains \\
\hline lever & every & loger & lower \\
\hline retard & regard & sport & report \\
\hline variable & available & libre & litre \\
\hline
\end{tabular}

Table $I V$. Examples of false friends in SDTES cognate matching.

Although these false friends can potentially hurt the misalignment detection algorithm in SDTES, their impact on the actual identification of misaligned pairs is not so significant. We extracted all the lines 
which match "--- pass cognates:" in the misalignment detection result file, and discovered that not many false friends are actually used for the cognate matching criterion in the misalignment detection process. Generally speaking, if two cognate words are not true friends, and if the text segments where they occur are not true translations of each other, chances are good that the misalignment would have been detected by the length criterion and the matching type criterion prior to the application of the cognates matching process (see Figure 8). In some cases, there are other cognate pairs that are checked before the false friend pair. Once a cognate match is found in one of these candidate cognate pairs, the aligned text segments are marked as pass. We also examined a few instances where the false friends are actually used as a decisive factor in the misalignment detection process and we found all of the aligned text segments to be true translations. It is often the case that one word of the false friend pair is the translation of another word in the text segment of the other language.

In alignment problem detection, for mismatches in numbers, the system would mark the pair as problem. But actually, the texts may have no translation and alignment problems at all. For example, for different phone numbers of contacts for different languages, the discrepancy in numbers does not mean that the sentences are misaligned or the translation is problematic. It is the right way to indicate that, for different languages, different phone numbers should be called. The same is true with the use of numbers indicating a particular year. When we say 2006 in French and say 'this year' in English, it becomes a 'problem' match because of different ways of expressing the same content meaning. But the lack of the number '2006' in English does not constitute a misalignment. Thus, the label 'problem' becomes problematic in this instance.

\section{Conclusion}

In this paper, we have described the general framework of SDTES, a system for the automatic extraction of translation pairs from web-based bilingual materials. 70,555 translation segments were extracted from the StatCan Daily data bank which was composed of the news release texts from 2004 to 2006 at Statistics Canada. The length-based statistical model and other algorithms were used together with the structural and textual features of the HTML texts for alignment of text segments and for detection of the misaligned parts. The aligned pairs were further filtered and formatted for easy portability into the translation memory 
systems, bilingual information retrieval databases and other natural language processing applications. The evaluation and results show that the automatic translation extraction system is a very suitable model for generating translation pairs from the type of web-based document of which Daily texts are an example. Introduction of the HTML structural features as major text block anchoring points prior to the utilization of the Gale-Church statistical method greatly enhanced the performance of the statistical parallel alignment and the capacity of thwarting massive misalignment. The SDTES system proves that textual features such as cognates and punctuation marks can play a very important role in aiding the misalignment detection process. The system introduces a two-step method of identifying cognates in bi-texts. The AMS search based on the candidate matching list produced by the K-vec algorithm performed very reliably and accurately. The major components of the system, with some parameters properly tuned, can be adapted for translation extraction of other web-based Canadian government publications. The extracted translations can be readily fed into translation memory systems, machine translation systems, cross-language information retrieval systems and other natural language processing systems.

\section{Acknowledgement}

Thanks go to the managers and staff of Statistics Canada at various levels: the Communications and Information Services Branch, the Communications and Library Services Division, and the Official Languages and Translation Division. They gave us full support for this research project. We are also indebted to Professor Andrew Brook and Professor Jim Davies of Carleton University, to Dr. Collin Baker of the International Computer Science Institute, to Professor Charles J. Fillmore of the University of California at Berkeley and ICSI, and to the anonymous reviewers of this paper, for their valuable comments and suggestions. 


\section{References}

Brown P F, Lai J C, Mercer R L (1991) Aligning sentences in parallel corpora. Proceedings, 29th Annual Meeting of the Association for Computational Linguistics, Berkeley, CA, pp 89-94

Callison-Burch C, Bannard C, Schroeder J (2005) A Compact Data Structure for Searchable Translation Memories. European Association for Machine Translation 2005 Conference

Chen A, Gey F (2001) Translation Term Weighting and Combining Translation Resources in Cross-Language Retrieval. TREC 2001

Chen J, Nie J Y (2000) Parallel Web Text Mining for Cross-Language IR. Proceedings of RIAO 2000: Content-Based Multimedia Information Access, Vol. 1, pp 62-78

Chen S (1996) Building Probabilistic Models for Natural Language. Ph.D. thesis, Harvard University, Cambridge, MA

Chesñevar C, Sabaté M, Maguitman A (2006) An Argument-based Decision Support System for Assessing Natural Language Usage on the Basis of the Web Corpus. International Journal of Intelligent Systems, Vol. 21, Issue 11, pp 1151-1180

Danielsson P, Mühlenbock K (2000) The Misconception of HighFrequency Words in Scandinavian Translation. AMTA 2000, pp 158168

Deng Y, Kumar S, Byrne W (2006) Segmentation and alignment of parallel text for statistical machine translation. Journal of Natural Language Engineering, 12(4), Cambridge University Press

Fung P, Church K W (1994) K-vec: A New Approach for Aligning Parallel Texts. Proceedings of the 15th International Conference on Computational Linguistics, Kyoto, Japan 
Gale W A, Church K W (1991) A program for aligning sentences in bilingual corpora. Proceedings of the 29th Annual Meeting of the Association for Computational Linguistics, Berkeley, CA. pp 177-184

Gey, F C, Chen A, Buckland M K, Larson R R (2002) Translingual vocabulary mappings for multilingual information access. In: SIGIR 2002, pp 455-456

Hutchins J (2005) Towards a definition of example-based machine translation. In: MT Summit X. workshop: Second workshop on example-based machine translation. Phuket, Thailand, pp 63-70

Inkpen D, Frunza O, Kondrak G (2005) Automatic Identification of Cognates and False Friends in French and English. Proceedings of RANLP'05, pp 251-257

Jutras J-M (2000) An Automatic Reviser, The TransCheck System. Proceedings of Applied Natural Language Processing, pp 127-134

Kay M, Röscheisen M (1993) Text-Translation Alignment. Computational Linguistics, 19(1): 121-142

Li K W, Yang Christopher C (2006) Conceptual analysis of parallel corpus collected from the Web. Journal of the American Society for Information Science and Technology, Vol. 57, Issue: 5, pp 632-644

McArthur T (eds) (1992) The Oxford Companion to the English Language. Oxford University Press, Oxford

Melamed I D (1999) Bitext Maps and Alignment via Pattern Recognition. Computational Linguistics, 25(1), 107-130

Moore, R C (2002) Fast and Accurate Sentence Alignment of Bilingual Corpora. AMTA, pp 135-144

Neumann S, Hansen-Schirra S (2005) The CroCo Project. Crosslinguistic corpora for the investigation of explicitation in translations. Proceedings of the Corpus Linguistics Conference, Birmingham, UK 
Pedersen T, Varma N (2002) K-vec++: Approach For Finding Word

Correspondences. Available online: http://www.d.umn.edu/

-tpederse/parallel.html. Cited 25 Aug 2007.

Resnik P (1999) Mining the Web for Bilingual Text. Proceedings of the 37th Annual Meeting of the Association for Computational Linguistics, College Park, MD. pp 527-534

Resnik P, Smith, Noah A (2003) The Web as a parallel corpus.

Computational Linguistics, 29 (3), 349-380

Ribeiro A, Dias G, Lopes G, Mexia J (2001) Cognates Alignment. In: Bente Maegaard (Ed) Proceedings of the Machine Translation Summit VIII (MT Summit VIII) - Machine Translation in the Information Age, Santiago de Compostela, Spain, pp 287-292

Sanchez-Villamil E, Santos-Anton S, Ortiz-Rojas S, Forcada M L (2006) Evaluation of Alignment Methods for HTML Parallel Text. LNCS 4139, p 280

Sigurbjörnsson B, Kamps J Rijke, de M (2005) EuroGOV: Engineering a Multilingual Web Corpus. Proceedings of CLEF 2005

Simard M, Foster G, Isabelle P (1992) Using Cognates to Align Sentences in Bilingual Corpora. Proceedings of the Fourth International Congress on Theoretical and Methodological Issues in Machine Translation (TMI 92), Montreal, Canada, pp 67-81

Simões A M, Almeida J J (2006) Combinatory Examples Extraction for Machine Translation. In: EAMT 11th Annual Conference. Oslo, Norway, pp 27-32

Tufis D., Barbu A M R Ion (2004) Extracting Multilingual Lexicons from Parallel Corpora. Computers and the Humanities, 38 (2), 163-189

Véronis J (2000a) Alignement de corpus multilingue. In: Ingénierie des langues, Traité IC2-Série Informatique et SI par Jean-Marie Pierrel, Èditions Hermes Science, Paris

Véronis J (2000b) From the Rosetta stone to the information society: a survey of parallel text processing. In: Véronis J (Ed) Parallel text 
processing: alignment and use of translation corpora, Kluwer Academic Publishers, pp 1-24

Wang J H, Jei-Wen Teng J W, Lu W H, Chien L F (2006) Exploiting the Web as the multilingual corpus for unknown query translation.

Journal of the American Society for Information Science and

Technology, Vol. 57, Issue: 5, pp 660-670

Wu D (1994) Aligning a parallel English-Chinese corpus statistically with lexical criteria. Proceedings of the 32nd Annual Meeting of the Association for Computational Linguistics, pp 80-87 